\title{
Asymmetry of metallic spectral lines in Cepheids
}

\author{
Michael Albrow , P.L. Cottrell \\ Mount John University Observatory, Department of Physics and Astronomy, \\ University of Canterbury, Christchurch, New Zealand
}

\begin{abstract}
A program of high resolution spectroscopic observations of Cepheids has been carried out at Mt John University Observatory for several years. Radial velocities and asymmetries have been measured for selected metallic lines using the method of Wallerstein et al (1992).

The line profiles show the largest asymmetry at phases of maximum inward velocity. The asymmetry at phases of maximum outward velocity is smaller and sometimes in the same direction as for the inward velocity maxima. Enhanced asymmetry is also noticed at phases where the bump appears on the radial velocity curve.

To date our models are unable to predict such behaviour. It is important that a satisfactory explaination for these observations be found so that the accuracy of Baade-Wesselink radius solutions for such stars can be assessed.
\end{abstract}
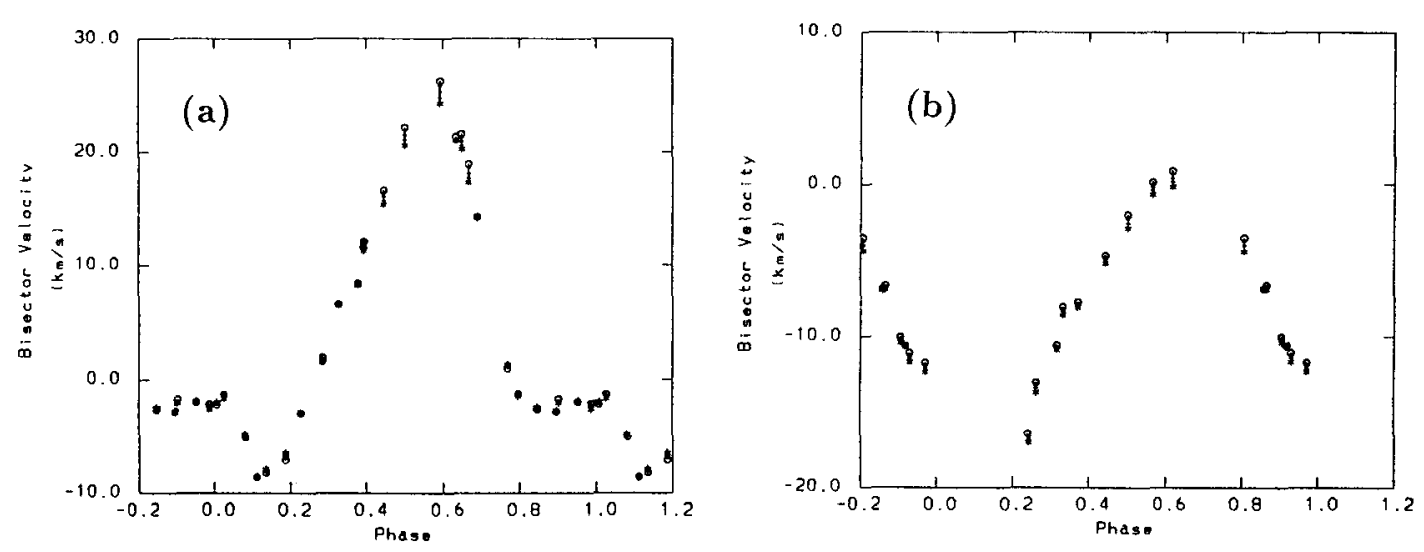

Figure 1 Radial velocities and asymmetries from the Fe I line at $6546 \AA$ for the Cepheids a) S Nor and b) Y Oph.

\section{References:}

Wallerstein,G., Jacobsen,T., Cottrell,P.L., Clark,M. \& Albrow,M., 1992, M.N.R.A.S. in press. 